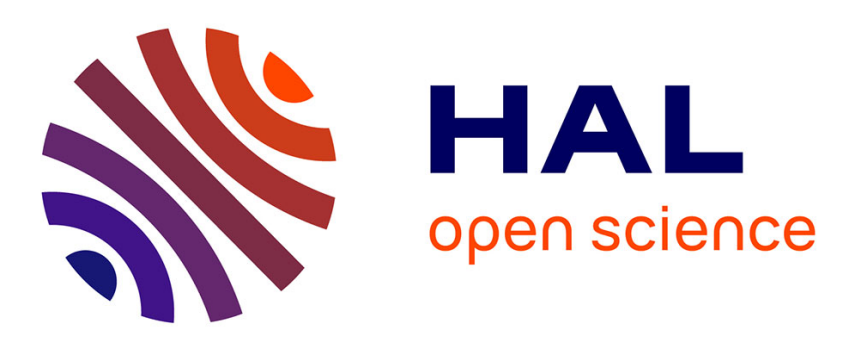

\title{
Disturbance energy transport and sound production in gaseous combustion
}

Michael J. Brear, Franck Nicoud, Mohsen Talei, Alexis Giauque, Evatt R.

Hawkes

\section{- To cite this version:}

Michael J. Brear, Franck Nicoud, Mohsen Talei, Alexis Giauque, Evatt R. Hawkes. Disturbance energy transport and sound production in gaseous combustion. Journal of Fluid Mechanics, 2012, 707, pp.53-73. 10.1017/jfm.2012.264 . hal-00789934

\section{HAL Id: hal-00789934 \\ https://hal.science/hal-00789934}

Submitted on 16 Sep 2013

HAL is a multi-disciplinary open access archive for the deposit and dissemination of scientific research documents, whether they are published or not. The documents may come from teaching and research institutions in France or abroad, or from public or private research centers.
L'archive ouverte pluridisciplinaire HAL, est destinée au dépôt et à la diffusion de documents scientifiques de niveau recherche, publiés ou non, émanant des établissements d'enseignement et de recherche français ou étrangers, des laboratoires publics ou privés. 


\title{
Disturbance energy transport and sound
}

\section{production in gaseous combustion}

\section{By MICHAEL J. BREAR ${ }^{1} \dagger$, FRANK NICOU D MOHSEN TALEI ${ }^{1}$, ALEXIS GIAUQUE}

AND EVATT R. HA W KES ${ }^{4}$

\author{
${ }^{1}$ Department of Mechanical Engineering, University of Melbourne, Parkville 3010, Australia \\ ${ }^{2}$ Université Montpellier II, I3M, Place E. Bataillon, 34095 Montpellier, France \\ ${ }^{3}$ Computational Aeroacoustics Department, Office National d'Etudes et de Recherches \\ Aerospatiales, Chatillon, France \\ ${ }^{4}$ School of Photovoltaic and Renewable Energy Engineering / School of Mechanical and \\ Manufacturing Engineering, University of New South Wales, Sydney 2052, Australia
}

(Received ?? and in revised form ??)

This paper presents an analysis of the energy transported by disturbances in gaseous combustion. It extends the previous work of Myers [J. Fluid Mech. 226 (1991) 383-400] and so includes non-zero mean flow quantities, large amplitude disturbances, varying specific heats and chemical non-equilibrium. This extended form of Myers' 'disturbance energy' then enables complete identification of the conditions under which the famous Rayleigh source term can be derived from the equations governing combusting gas motion. These are: small disturbances in an irrotational, homentropic, non-diffusive (in terms of species, momentum and energy) and stationary mean flow at chemical equilibrium. Under these assumptions, the Rayleigh source term becomes the sole source term in a conservation equation for the classical acoustic energy.

It is also argued that the exact disturbance energy flux should become an acoustic energy flux in the far-field surrounding a (reacting or non-reacting) jet. In this case, 
the volume integral of the disturbance energy source terms are then directly related to the area averaged, far-field sound produced by the jet. This is demonstrated by closing the disturbance energy budget over a set of aeroacoustic, Direct Numerical Simulations (DNS) of a forced, low Mach number, laminar, premixed flame. These budgets show that several source terms are significant, including those involving the mean flow and entropy fields. This demonstrates that the energetics of sound generation in these simulations cannot be examined by considering the Rayleigh source term alone.

\section{Introduction}

Combustion generated sound and combustion system stability are closely related problems. In many combustion systems, instability is initiated by the reflection of combustion generated sound, which in turn further excites the mechanisms by which the sound was first generated. Entropy and equivalence ratio disturbances can also play a role. The resulting 'thermoacoustic instability' can lead to very high amplitude sound production and, in the worst cases, catastrophic mechanical failure of the system.

Research in combustion generated sound and combustion stability has received sustained attention in both the academic and industrial communities over the last fifty years in particular. During this time, the literature has grown enormously, and now spans numerous applications including rockets, afterburners, gas turbines and industrial burners (Putnam 1971; Flandro 1985; Bloxsidge et al. 1988; Poinsot \& Veynante 2001; Culick 2001; Candel 2002; Dowling \& Stow 2003; Schwarz \& Janicka 2009). This sustained effort is in large part due to gas turbine manufacturers still relying heavily on in-situ testing and tuning of the complete, operating device to avoid instability. In the authors' view, this continued reliance on testing has several causes, including an incompleteness in 
our fundamental understanding of all the mechanisms involved in combustion generated sound.

The so-called 'Rayleigh term' is commonly used to explain the energetics of sound production by heat addition, e.g. (Putnam 1971). Whilst Lord Rayleigh (1878) first stated this term in prose form, it is usually expressed mathematically as $\overline{p^{\prime} \omega^{\prime}}$ where $p^{\prime}$ and $\omega^{\prime}$ are harmonic disturbances in the static pressure and heat release rate at a point in space respectively, and the overbar $\overline{()}$ denotes the time average over one cycle. This term states that net sound is produced when the relative phase of the pressure and heat release disturbances are such that the time average of their product is positive. Despite the Rayleigh term being identified well over a century ago, Nicoud \& Poinsot (2005) suggested that it is still at the very least unclear under what conditions this criterion can be derived from the equations governing combusting fluid motion. The earlier works of Chu (1956, 1965), Bloxsidge et al. (1988) and Dowling (1997) attempted to show precisely this, using progressively more general definitions of acoustic or disturbance energies that have appeared over the last fifty or so years.

In combusting flows, any conservation equation that describes the energy contained in flow disturbances should at least initially consider the effects of a non-zero mean flow. Ignoring mean flow causes a conceptual issue since any fluctuations in heat release must be around a zero mean heat release, and so involve cooling of the flow at some instants. This issue is discussed further later in this paper. The energies defined by Morfey (1971), Doak (1989) and Myers (1991) include a mean flow, and hence the works by Bloxsidge et al. (1988) and Dowling (1997), which are based on Morfey (1971), do not suffer from this conceptual problem.

Morfey (1971) considered a viscous, heat conducting fluid, and first split the disturbance velocity field into irrotational and solenoidal components which were defined as 
the acoustic and unsteady vortical motions respectively. He then applied the definitions of the acoustic energy density and flux proposed by Cantrell \& Hart (1964) for inviscid, non-heat conducting flows to his acoustic field. Any resulting entropy disturbances in Morfey's analysis were then shifted into the source term. However, the definition of all irrotational velocity disturbances as acoustic can be problematic in reacting flows, which can, for example, feature irrotational, non-acoustic velocity disturbances due to heat addition (e.g. Talei et al. $(2011,2012))$.

The energy defined by Doak (1989) potentially addresses this issue by splitting the irrotational component of the momentum density disturbance $(\rho \mathbf{u})^{\prime}$ into acoustic and 'thermal' components, defined as isentropic and isobaric disturbances respectively. However, this may once again be problematic in combustion. Irrotational 'thermal' disturbances are not necessarily isobaric, for example during large amplitude thermoacoustic instability (e.g. Flandro (1985); Culick (2001); Dowling \& Stow (2003)), and irrotational acoustic disturbances are not in general isentropic in a region of heat addition (e.g. Karimi et al. $(2008,2010))$.

Further, combustion also interacts with the mean and fluctuating vorticity and entropy fields, and in many devices does not occur at low Mach number, inferring that separation of acoustic and convective scales is difficult. An added problem is that many disturbances are not small in combusting flows. For example, the heat release, temperature and entropy disturbances at a point in space can have similar amplitude as their mean values as a flame moves back and forth past that point. As for inhomogeneous flows more generally (e.g. Goldstein (2005)), the unambiguous separation of radiating and non-radiating disturbances is therefore a particular challenge in combustion.

In studying the energetics of combusting flows, it may therefore be advantageous to use an exact energy equation that does not attempt to separate sound from other distur- 

bances and which permits a non-zero mean flow. This appears to be the case only with the energy defined by Myers (1991). Myers allowed entropy disturbances to remain in both the energy density and flux terms, and did not split the velocity field in any way. Myers' equation was consistent with those developed earlier by Chu (1965) and Pierce (1981) for zero mean flow. However, because the energy density and flux terms contained entropy disturbances, the resulting energies are not acoustic and are more properly called a 'disturbance energy' (Chu (1965); Myers (1991)).

Care must therefore always be taken when examining fluid flows with the energy defined by Myers (1991), since it does not distinguish between energy transport by different types of disturbance. Further, not all terms in its most general form are positive definite or second order in the disturbances; undesirable features that it shares with the acoustic energy of Cantrell \& Hart (1964). Thus, Myers' disturbance energy becomes most useful when it can be simplified to energies that have a more physical meaning. In the present study, this is done by time averaging the disturbance energy conservation equation around a stationary base flow, and applying Gauss' Law to a precisely defined far-field. This results in a time averaged disturbance energy balance whose flux can be interpreted as sound.

This paper first extends the work of Myers (1991) to gaseous combustion by including the transport of multiple species, chemical non-equilibrium and heat release terms into his exact disturbance energy corollary. This exact equation is then approximated to second order, demonstrating the existence of several source terms other than the Rayleigh term. It is then demonstrated that Myers' exact disturbance energy flux becomes the acoustic energy flux in the far-field surrounding a jet by closing the budget of this extended disturbance energy over a series of Direct Numerical Simulations (DNS) of a forced, low Mach number, laminar, premixed flame. These budgets show that several source 
terms are significant, demonstrating that the energetics of sound generation cannot be examined by considering the Rayleigh term alone.

\section{Theory}

The equations of motion for gaseous combustion are first cast in a form consistent with that in Myers (1991). These equations ignore body forces.

\subsection{Equations of motion}

\subsubsection{Momentum equation}

The momentum equation can be derived from a more common form,

$$
\frac{\partial u_{j}}{\partial t}+u_{i} \frac{\partial u_{j}}{\partial x_{i}}+\frac{1}{\rho} \frac{\partial p}{\partial x_{j}}=\frac{1}{\rho} \frac{\partial \tau_{i j}}{\partial x_{i}},
$$

where $u_{i}$ is the component of the velocity vector $\mathbf{u}$ in direction $x_{i}, \rho$ is the density, $p$ is the static pressure and $\tau_{i j}$ is the $i j^{t h}$ component of the viscous stress tensor. It is first noted that the convective acceleration can be written as

$$
u_{i} \frac{\partial u_{j}}{\partial x_{i}}=\nabla\left(\frac{1}{2}|u|^{2}\right)+\boldsymbol{\xi} \times \mathbf{u},
$$

where $\boldsymbol{\xi}=\boldsymbol{\nabla} \times \mathbf{u}$ is the vorticity. Gibbs' equation for a reacting mixture of $n$ species can be written per unit mass as (Williams 1985)

$$
d e=T d s-p d\left(\frac{1}{\rho}\right)+\sum_{k=1}^{n} g_{k} d Y_{k},
$$

where $e$ is the internal energy, $s$ is the entropy whilst $g_{k}$ and $Y_{k}$ are the Gibbs free energy and mass fraction of the $k^{t h}$ species. It is noted that $g_{k}$ is equal to the chemical potential $\mu_{k}$ of that species Williams (1985). Gibbs' equation and the definition of the enthalpy $h=e+p / \rho$, can be combined to show that

$$
\frac{\boldsymbol{\nabla} \boldsymbol{p}}{\rho}=\boldsymbol{\nabla} h-T \nabla s-\sum_{k=1}^{n} g_{k} \nabla Y_{k} .
$$


Combined with the momentum equation and the vector form of the convective acceleration above, this results in a modified form of Crocco's theorem for an unsteady, viscous and combusting gas,

$$
\frac{\partial \mathbf{u}}{\partial t}+\boldsymbol{\zeta}+\nabla H-T \nabla s=\boldsymbol{\psi}+\boldsymbol{\psi}^{*}
$$

where $\boldsymbol{\zeta}=\boldsymbol{\xi} \times \mathbf{u}, \boldsymbol{\psi}=(1 / \rho) \partial \tau_{i j} / \partial x_{i}$ and $\boldsymbol{\psi}^{*}=\sum_{k=1}^{n} g_{k} \boldsymbol{\nabla} Y_{k}$. It is relatively straight forward to show that the enthalpy, entropy and Gibb's free energy in this equation can be considered all as either sensible or total chemical quantities.

\subsubsection{Energy equation}

The energy equation can be written as (e.g. Poinsot \& Veynante (2001))

$$
\frac{\partial}{\partial t}(\rho H-p)+\nabla \cdot(\mathbf{m} H)-\mathbf{m} \cdot \boldsymbol{\psi}=T Q
$$

where $\mathbf{m}=\rho \mathbf{u}$ is the mass flux vector, $H$ is the sensible stagnation enthalpy and $T$ is the static temperature. The term $Q$ includes the thermal and species diffusion flux vector q, viscous dissipation $\phi$ and combustion heat release $\omega$ terms. Assuming Fourier's law of conduction with thermal conductivity $\lambda, Q$ can be written as

$$
Q=\frac{1}{T}(\nabla \cdot \mathbf{q}+\phi+\omega)
$$

where

$$
\boldsymbol{\nabla} \cdot \mathbf{q}=-\boldsymbol{\nabla} \cdot(\lambda \boldsymbol{\nabla} T)+\rho \boldsymbol{\nabla} \cdot\left(\sum_{i=1}^{n} h_{k} Y_{k} \boldsymbol{V}_{\boldsymbol{k}}\right), \phi=\tau_{i j} \frac{\partial u_{j}}{\partial x_{i}}, \omega=-\sum_{i=1}^{n} \Delta h_{f, k}^{0} \omega_{k},
$$

and $\boldsymbol{V}_{\boldsymbol{k}}$ is the diffusion velocity of the $k^{\text {th }}$ species.

\subsubsection{Entropy transport equation}

The entropy transport equation also starts with Gibbs' equation, now written with the substantial derivative,

$$
\rho T \frac{D s}{D t}=\rho \frac{D e}{D t}-\frac{p}{\rho} \frac{D \rho}{D t}-\rho \sum_{k=1}^{n} g_{k} \frac{D Y_{k}}{D t} .
$$


Here, the first term on the right hand side is the energy equation in terms of the internal energy. The second term can be shown to be equal to $-p(\boldsymbol{\nabla} \cdot \mathbf{u})$. Application of the species transport equation to the last term then finally yields the sensible entropy transport equation

$$
\frac{D(\rho s)}{D t}=Q+Q^{*}
$$

where $Q$ was defined in equation 2.6 and

$$
Q^{*}=-\left(\sum_{k=1}^{n} g_{k} \omega_{k}+\sum_{k=1}^{n} g_{k} \boldsymbol{\nabla} \cdot\left(\rho \boldsymbol{V}_{\boldsymbol{k}} Y_{k}\right)\right) / T
$$

All the equations required for extending the equations of Myers (1991) to gaseous combustion are now in an appropriate form.

\subsection{Extended form of Myers' exact energy corollary around a steady base flow}

Myers (1991) commenced his derivation of his exact disturbance energy conservation equation from statements of mass conservation, momentum transport, energy conservation and entropy transport. Transport of $(n-1)$ species is added in the present analysis which, together with mass conservation, guarantees conservation of the $n^{\text {th }}$ species. Using the derivations presented in Section 2.1, the complete equations of motion are

$$
\begin{aligned}
\frac{\partial \rho}{\partial t}+\nabla \cdot \mathbf{m} & =0 \\
\sum_{k=1}^{n-1}\left[\frac{\partial \rho Y_{k}}{\partial t}+\nabla \cdot\left(\mathbf{m} Y_{k}\right)\right. & \left.=\omega_{k}-\nabla \cdot\left(\rho \mathbf{V}_{\mathbf{k}} Y_{k}\right)\right], \\
\frac{\partial \mathbf{u}}{\partial t}+\boldsymbol{\zeta}+\nabla H-T \nabla s & =\boldsymbol{\psi}+\boldsymbol{\psi}^{*}, \\
\frac{\partial}{\partial t}(\rho H-p)+\nabla \cdot(\mathbf{m} H)-\mathbf{m} \cdot \boldsymbol{\psi} & =T Q \\
\frac{\partial \rho s}{\partial t}+\nabla \cdot(\mathbf{m} s) & =Q+Q^{*}
\end{aligned}
$$

Equations 2.12 to 2.16 have steady solution,

$$
\nabla \cdot \mathbf{m}_{\mathbf{0}}=0
$$




$$
\begin{aligned}
\sum_{k=1}^{n-1}\left[\nabla \cdot\left(\mathbf{m}_{\mathbf{0}} Y_{k 0}\right)\right. & \left.=\omega_{k 0}-\nabla \cdot\left(\rho_{0} \mathbf{V}_{\mathbf{k} \mathbf{0}} Y_{k 0}\right)\right], \\
\boldsymbol{\zeta}_{\mathbf{0}}+\boldsymbol{\nabla} H_{0}-T_{0} \boldsymbol{\nabla} s_{0} & =\boldsymbol{\psi}_{\mathbf{0}}+\boldsymbol{\psi}_{\mathbf{0}}{ }^{*} \\
\boldsymbol{\nabla} \cdot\left(\mathbf{m}_{\mathbf{0}} H_{0}\right)-\mathbf{m}_{\mathbf{0}} \cdot \boldsymbol{\psi}_{\mathbf{0}} & =T_{0} Q_{0} \\
\boldsymbol{\nabla} \cdot\left(\mathbf{m}_{\mathbf{0}} s_{0}\right) & =Q_{0}+Q_{0}^{*} .
\end{aligned}
$$

Following the approach of Myers (1991), equations 2.12, 2.13 and 2.16 can be multiplied by $\left(H_{0}-T_{0} s_{0}-g_{0}\right), g_{k 0}$ and $T_{0}$ respectively and added to the scalar product of equation 2.14 and $\mathbf{m}_{\mathbf{0}}$. This sum is subtracted from equation 2.15 and, after considerable algebra that mirrors that detailed in Myers (1991) and which utilises equations 2.17 to 2.21, results in an exact conservation equation for a 'disturbance energy' of the form

$$
\frac{\partial E}{\partial t}+\nabla \cdot \mathbf{W}=D
$$

In equation 2.22, the disturbance energy density $E$ and flux vector $\mathbf{W}$ terms are respectively

$$
E=\rho\left(H^{\prime}-T_{0} s^{\prime}\right)-\mathbf{m}_{\mathbf{0}} \cdot \mathbf{u}^{\prime}-p^{\prime}-\rho \sum_{k=1}^{n-1} g_{k 0} Y_{k}^{\prime}
$$

and

$$
\mathbf{W}=\mathbf{m}^{\prime}\left(H^{\prime}-T_{0} s^{\prime}\right)+\mathbf{m}_{\mathbf{0}} T^{\prime} s^{\prime}
$$

where ()$^{\prime}=()-()_{0}$ is the disturbance and ()$_{0}$ is the steady value. The exact source term $D$ is

$$
D=D_{\zeta}+D_{s}+D_{Q}+D_{Q^{*}}+D_{\psi}+D_{\psi^{*}}+D_{Y_{k}}
$$

where

$$
\begin{gathered}
D_{\zeta}=-\mathbf{m}^{\prime} \cdot \boldsymbol{\zeta}^{\prime}, D_{s}=-\mathbf{m}^{\prime} \cdot\left(s^{\prime} \nabla T_{0}\right)+s^{\prime} \mathbf{m}_{\mathbf{0}} \cdot \nabla T^{\prime}, D_{Q}=T^{\prime} Q^{\prime}, \\
D_{Q^{*}}=T^{\prime} Q^{* \prime}, D_{\psi}=\mathbf{m}^{\prime} \cdot \boldsymbol{\psi}^{\prime}, D_{\psi^{*}}=\mathbf{m}^{\prime} \cdot \boldsymbol{\psi}^{* \prime}, D_{Y_{k}}=g^{\prime} \boldsymbol{\nabla} \cdot \mathbf{m}^{\prime}+\sum_{k=1}^{n-1} g_{k}^{\prime} \Omega_{k}^{\prime},
\end{gathered}
$$

and $\Omega_{k}=\omega_{k}-\nabla \cdot\left(\rho \mathbf{V}_{\mathbf{k}} Y_{k}\right)-\nabla \cdot\left(\mathbf{m} Y_{k}\right)$. Neglecting viscous stress, viscous dissipation, 
thermal diffusion and species diffusion terms, several terms in equation 2.25 can also be simplified to

$$
Q=\frac{\omega}{T}, Q^{*}=-\sum_{k=1}^{n-1} g_{k} \omega_{k} / T, \psi=0,
$$

with the other terms defined as above.

2.3. Extended form of Myers' exact energy corollary around an unsteady base flow Rather than the steady equations 2.17 to 2.21 , the time average of an unsteady flow can also be chosen as the base flow. The resulting energy corollary contains new terms, but can be used for post-processing data when the equivalent steady flow is not known, and simplifies significantly when time averaged. Appendix A contains the extended form of the exact disturbance energy corollary with an unsteady base flow, and this form of the corollary is used to analyse numerical simulations of combustion generated sound in Section 5 .

\subsection{Second order corollary and chemical equilibrium}

A second order corollary may not be accurate since, as discussed in the Introduction, combusting flows do not usually feature small disturbances. Nonetheless, a second order disturbance energy corollary must first be found in order to show how the extended form of the disturbance energy corollary simplifies to other disturbance or acoustic energy conservation equations.

A derivation of one such second order energy corollary is given in Appendix B, which can be summarised as

$$
\frac{\partial E_{2}}{\partial t}+\nabla \cdot \mathbf{W}_{2}=D_{2}
$$

In the exact analysis of the preceeding sections, $g_{0}$ and $g_{k 0}$ multiplied by equations 2.12 and 2.13 respectively are additional to the original approach by Myers (1991), and result in exactly second order flux $\boldsymbol{W}$ and source terms $D$. Nonetheless, approximating the 

energy density in the exact equation 2.22 to its second order equivalent $E_{2}$ in equation 2.26 is substantially more complex since there are now $(n+1)$ independent variables in which to expand the exact equation.

As required, the disturbance energy density given in equation B 3 reduces to that proposed by Myers (1991) for a gas containing a single species. Nonetheless, the terms involving mass fraction disturbances are very complex and are not positive definite in general fluid flows. It is therefore difficult to see how such an expression can be useful. Fortunately, further use of the second order corollary in this paper does not involve analysis of this term.

Previous studies of acoustic or disturbance energy transport in combustion usually assume chemical equilibrium (e.g. Bloxsidge et al. (1988), Chu (1956), Dowling (1997), Flandro (1985), Nicoud \& Poinsot (2005)). In this case the terms involving the Gibbs free energy in equations $2.14,2.16$ are zero and equation 2.13 is no longer required, simplifying equation 2.22 and its second order equivalent, equation 2.26. For a non-diffusive flow, the following second order energy density $E_{2}$, flux $\mathbf{W}_{\mathbf{2}}$ and source terms $D_{2}$ then result.

$$
\begin{gathered}
E_{2}=\frac{p^{\prime 2}}{2 \rho_{0} c_{0}^{2}}+\frac{1}{2} \rho_{0} u^{\prime 2}+\rho^{\prime} \mathbf{u}_{\mathbf{0}} \cdot \mathbf{u}^{\prime}+\frac{\rho_{0} T_{0} s^{\prime 2}}{2 c_{p 0}} \\
W_{2}=\left(p^{\prime}+\rho_{0} \mathbf{u}_{\mathbf{0}} \cdot \mathbf{u}^{\prime}\right)\left(\mathbf{u}^{\prime}+\frac{\rho^{\prime}}{\rho_{0}} \mathbf{u}_{\mathbf{0}}\right)+\mathbf{m}_{\mathbf{0}} T^{\prime} s^{\prime}
\end{gathered}
$$

and

$$
D_{2}=\mathbf{m}^{\prime} \cdot \zeta^{\prime}-s^{\prime} \mathbf{m}^{\prime} \cdot\left(\nabla T_{0}\right)+s^{\prime} \mathbf{m}_{\mathbf{0}} \cdot\left(\boldsymbol{\nabla} T^{\prime}\right)+\left(\frac{\omega^{\prime} T^{\prime}}{T_{0}}-\frac{\omega_{0} T^{2}}{T_{0}^{2}}\right) .
$$

It is noted that the equilibrium results $\left(H^{\prime}-T_{0} s^{\prime}\right)=p^{\prime} / \rho_{0}+\mathbf{u}_{\mathbf{0}} \cdot \mathbf{u}^{\prime}$ and $p^{\prime}=c_{0}^{2} \rho_{0} s^{\prime} / c_{p 0}+$ $c_{0}^{2} \rho^{\prime}$ have been used to derive equations 2.27 and 2.28. The terms containing the steady $\omega_{0}$ and unsteady $\omega^{\prime}$ heat release in equation 2.29 are additional to Myers (1991) for this case of a non-diffusive flow at chemical equilibrium. 


\section{Discussion of the theoretical results}

\subsection{Comparison to prior works}

As required, equation 2.26 simplifies to existing acoustic energy conservation equations under the condition of homentropic flow (i.e. $\nabla s=0$ everywhere). The second order energy density $E_{2}$ and flux $\mathbf{W}_{\mathbf{2}}$ terms then become those defined by Cantrell \& Hart (1964) for acoustic propagation in a non-stationary medium. In this case only the vortical source terms remain and represent the acoustic energy generated by unsteady vortical flow if it is assumed that sound features irrotational velocity disturbances (e.g. Dowling (1997))

Nicoud \& Poinsot (2005) derived a second order equation for the disturbance energy in a combusting flow assuming chemical equilibrium. Assuming chemical equilibrium for the present set of second order equations, as shown in equations 2.27 to 2.29 , the differences in the second order energy density and flux terms arise primarily due to Nicoud and Poinsot's assumption of zero mean flow quantities. Another, less significant difference is in the last term in the energy density, where Nicoud \& Poinsot (2005) assumed calorific perfection. Myers (1991) did not assume this, and so linear disturbances in entropy properly include the specific heat of the steady state $c_{p 0}$.

Equations 2.25 and 2.29 are source terms for gaseous combustion, with some of the second order terms present in the source term of Nicoud \& Poinsot (2005). It is interesting to note that the terms containing entropy disturbances may interfere, so care must be taken in understanding their combined effects. Equation 2.29 also shows a source term $\omega_{0} T^{2} / T_{0}^{2}$. This term is later shown to have significant magnitude, is positive definite, and can interfere with the term $\omega^{\prime} T^{\prime} / T_{0}$. This term was studied in detail in an earlier work (Karimi et al. (2008)), and showed that disturbance and acoustic energy can be created or destroyed in the absence of unsteady heat addition or any form of diffusion. 
Finally, the term $D_{2}$ in this paper and in Nicoud \& Poinsot (2005) both contain a term $\omega^{\prime} T^{\prime} / T_{0}$, from which the Rayleigh term arises under certain circumstances. This term only becomes the Rayleigh term $\omega^{\prime} p^{\prime}$ for homentropic flows, since then $p^{\prime}$ is proportional to $T^{\prime}$. Of course, combustion is not homentropic.

\subsection{Reducing the disturbance energy corollary to an equation with the Rayleigh term}

The complete set of conditions under which the equations governing combusting gas motion reduce exactly to an equation with the Rayleigh term as the sole source term can now be stated. Such flows must be irrotational, non-diffusive (in terms of species, momentum and energy), homentropic, without mean flow, at chemical equilibrium and feature only small disturbances. In this case, equation 2.26 reduces to a statement of conservation of the classical acoustic energy with a single source term, i.e.

$$
\frac{\partial}{\partial t}\left(\frac{p^{\prime 2}}{2 \rho_{0} c_{0}^{2}}+\frac{\rho_{0} u^{\prime 2}}{2}\right)+\nabla \cdot\left(p^{\prime} \mathbf{u}^{\prime}\right)=\frac{\left(\gamma_{0}-1\right)}{\gamma_{0} p_{0}} p^{\prime} \omega^{\prime}
$$

where $\gamma_{0}$ is the steady ratio of specific heats.

These conditions render the Rayleigh term conceptually problematic for combusting flows, particularly because of the mean heat release and entropy gradients in these flows. The requirement that disturbances in heat addition $\omega^{\prime}$ must be around a zero mean $\omega_{0}=0$ infers that at some instants combustion is cooling the flow. By permitting nonzero mean flow quantities, Myers (1991) permits a mean flux, and so can accommodate a positive mean heat release rate $\omega_{0}$ around which disturbances $\omega^{\prime}$ can occur without ever cooling the domain. However, once a mean flow is permitted, unsteady heat addition also generates entropy disturbances (e.g. Karimi et al. $(2008,2010)$ ), further violating the conditions required to derive the Rayleigh term. Of course, violation of these conditions might be tolerated if these effects are small. However, results presented later in this paper suggest that this is not the case, even for low Mach number combustion. 


\subsection{Application to sound generation by jet flows}

Following arguments put forward by Doak (1989), the acoustic intensity in the far-field surrounding a jet can be determined by integrating equation 2.22 over a volume $V$ surrounding the jet, out to an as yet undefined 'far-field', and then applying Gauss' theorem. For a time stationary flow, it follows that $\int_{V} \overline{(\partial E / \partial t)} d V=0$ and so

$$
\int_{A} \overline{\mathbf{W}} \cdot d \mathbf{A}=\int_{V} \bar{D} d V
$$

where $\overline{()}$ denotes the time average (and not the steady flow).

As equation $\mathrm{B} 4$ shows, the exact flux term $\mathbf{W}$ in equation 3.2 becomes the acoustic energy flux of Cantrell \& Hart (1964) if the far-field is defined as having negligible entropy and mass fraction disturbances. This, in turn, reduces to the classical acoustic energy flux $p^{\prime} \mathbf{u}^{\prime}$ if mean flow effects are negligible. The absence of significant entropy and mass fraction disturbances is a reasonable definition of the far-field, since these disturbances should decay much more quickly than sound due to diffusion. Under this assumption, the disturbance energy balance in equation 3.2 becomes

$$
\int_{A} \overline{\left(p^{\prime}+\rho_{0} \mathbf{u}_{\mathbf{0}} \cdot \mathbf{u}^{\prime}\right)\left[\mathbf{u}^{\prime}+\left(\rho^{\prime} / \rho_{0}\right) \mathbf{u}_{\mathbf{0}}\right]} \cdot d \mathbf{A}=\int_{V} \bar{D} d V
$$

for non-zero mean flow in the far-field and

$$
\int_{A} \overline{p^{\prime} \mathbf{u}^{\prime}} \cdot d \mathbf{A}=\int_{V} \bar{D} d V
$$

if far-field, mean flow effects are negligible.

Equations 3.3 and 3.4 give the area and time averaged acoustic energy flux in the farfield. They do not require the quantities to be constant over the area $A$. However, they do not show where inside the domain the sound is generated and do not separate the radiating and non-radiating components of the source terms at a point in space inside the volume $V$. Nonetheless, equation 3.3 expresses the contribution of the volume integral of the exact source terms to the area averaged, far-field sound. 
It is also noted that in many devices with a confined flame, this definition of the farfield may not be reached since significant entropy and mass fraction disturbances can exist up to the combustor boundaries. In this case, the disturbance energy flux does not reduce to an acoustic energy flux within the combustor, and the above approach does not give insight into the sound generation problem.

\section{Numerical methods}

The DNS results in this paper use a modified form of the code NTmix which features a 6th order compact scheme for spatial derivatives, combined with a 3rd order RungeKutta time integrator (Cuenot et al. (1997)). NTmix has been used extensively to study laminar and turbulent combustion (e.g. Poinsot \& Lele 1992; Baum 1994; Bourlioux et al. 2000), as well as non-reactive flows (Corjon \& Poinsot 1995, 1997).

A 2D slot flame is modelled in the present problem, with a schematic of the computational domain shown in Figure 1. The governing equations were discretised into 1021 streamwise nodes from $x=0$ to $32 L$ and 251 transverse nodes from $y=0$ to $4 L$, where $L$ is the half width in the slot flame. A non-uniform grid in both directions was set up such that the flame structure could be captured without compromising the far-field behaviour. Extensive grid independence studies were conducted to ensure proper resolution of both the flame and the acoustics. At least 6 nodes resolved the flame thickness at all times, and close quantitative agreement with the flame dynamics reported by Poinsot \& Lele (1992) were observed as one of several test cases.

Figure 1 features four boundary conditions. The velocity, temperature and unburnt mass fraction are imposed at the inlet using tanh profiles, resulting in a reflecting boundary condition. Along this inlet boundary and outside the flame, the velocity, temperature and unburnt mass fraction were $0, T_{b}$ and 0 respectively. A symmetry boundary condi- 


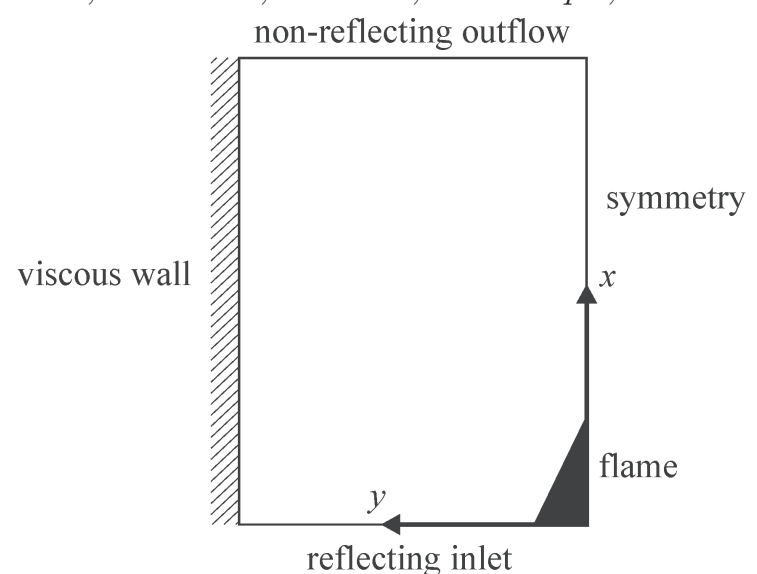

FiguRE 1. Schematic of computational domain (not to scale)

tion is used on the jet centreline, with a viscous wall boundary condition on the opposing wall. The outflow boundary is modelled with non-reflecting boundary conditions (Yoo et al. (2005); Lodato et al. (2008)).

In these simulations, the heat release rate from combustion $\omega$ defined in equation 2.6 for a mixture of $n$ species is now treated in a simplified form for single step chemistry,

$$
\omega=\Lambda \rho_{u} c_{p} Y_{u}\left(T_{b}-T_{u}\right) \exp \left(\frac{-\beta(1-\Theta)}{1-\alpha(1-\Theta)}\right),
$$

where

$$
\Lambda=B_{0} \exp \left(\frac{-\beta}{\alpha}\right), \Theta=\left(T-T_{u}\right) /\left(T_{b}-T_{u}\right),
$$

The terms $\rho_{u}, c_{p}$ and $Y_{u}$ in equation 4.1 are the unburnt mixture density, mixture specific heat (assumed constant) and unburnt mass fraction respectively, whilst $B_{0}$ is the preexponential factor, $\Theta$ is the reduced temperature, $T_{u}$ is the unburnt gas temperature and $T_{b}$ is the burnt gas temperature. The two parameters $\alpha$ and $\beta$ determine the flame heat release and activation temperature respectively (Poinsot \& Veynante (2001)).

The following non-dimensional parameters are specified.

$$
R e=\left(\frac{c_{i n} L}{\nu}\right)=2000, \operatorname{Pr}=\left(\frac{c_{p} \mu}{k}\right)=0.75, L e=\frac{S c}{\operatorname{Pr}}=1,
$$




$$
D a=\left(\frac{D \Lambda}{S_{L}^{2}}\right), M_{S_{L}}=\left(S_{L} / c_{i n}\right)=0.01, M_{j}=\left(\bar{u}_{i n} / c_{i n}\right)=0.04
$$

where $R e$ is the Reynolds number, $c_{i n}$ is the jet sonic velocity, $L$ is the reference length (the jet half width), $\nu$ is the kinematic viscosity, $\operatorname{Pr}$ is the Prandtl number, $\mu$ is the dynamic viscosity, $k$ is the thermal conductivity, Le is the Lewis number, $D a$ is the Damkohler number and $M_{S_{L}}$ and $M_{j}$ are Mach numbers based on the laminar flame speed $S_{L}$ and jet mean flow speed $u_{i} n$. The inflow velocity was varied sinusoidally over a wide range of forcing frequencies at $25 \%$ of the mean inflow velocity, with the forcing frequency represented as a Strouhal number

$$
S t=\left(f L / c_{i n}\right)
$$

where $f$ is the frequency of excitation. In all simulations the burnt to unburnt temperature ratio was $T_{b} / T_{u}=4$.

\section{Discussion of the numerical results}

Figure 2 shows instantaneous images of the temperature field surrounding two sinusoidally forced slot flames. The high $S t$ case is effectively stationary; there are very small oscillations in the flame surface during the forcing period. The low $S t$ case in Figure 2 shows a strongly wrinkled flame. Experimental studies of forced premixed laminar flames commonly demonstrate a qualitatively similar dynamic response to flame inlet velocity forcing (e.g. Schuller et al. (2003); Karimi et al. (2009)). In these cases, flame surface oscillations tend to zero amplitude as $S t$ increases, with $S t \sim 0.1-0.2$ being typical of the forcing frequencies at which these surface disturbances start to become small.

The wrinkled, low $S t$ case in Figure 2 also features so-called 'flame pinch-off' and 'flame island burn-out' annihilation events during each forcing period. This behaviour has also been observed by Schuller et al. (2003) and Karimi et al. (2009) in experimental studies 
i) ii)

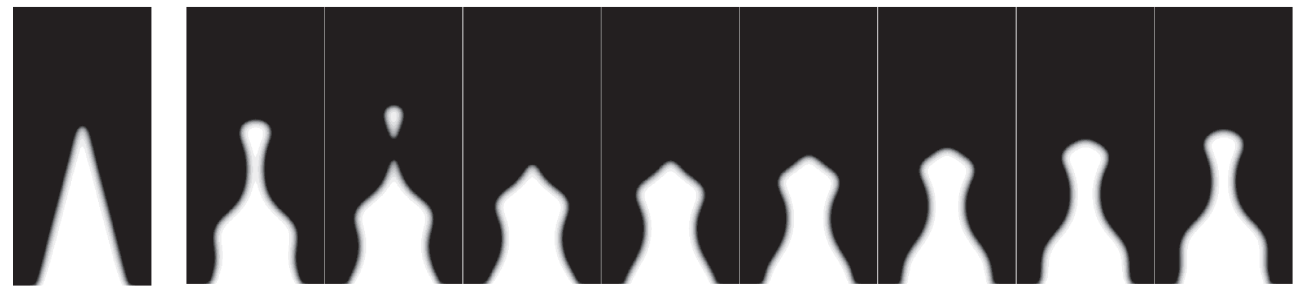

FiguRE 2. Instantaneous field of mass fraction burnt thesholded at $50 \%$ for i) $S t=1$ and ii)

$$
S t=0.02
$$

of forced, premixed laminar flames, and these annihilation events have been examined by the authors in detail in Talei et al. (2011).

Figure 3 shows the dilatation field $\boldsymbol{\nabla} \cdot \mathbf{u}$ at instants during one forcing period for a case where these annihilation events are strong. The dilatation and the pressure are related by a combination of the momentum and energy equations, such that for $\overline{\mathbf{u}}_{\infty}=0$,

$$
\frac{\partial p}{\partial t}+\rho_{\infty} c_{\infty}^{2} \nabla \cdot \mathbf{u}=0
$$

where the subscript $\infty$ refers to the variables in the far-field. The dilatation is commonly used in aero-acoustic studies to identify sound, e.g. Colonius et al. (1997). In the present study, the dilatation has the added, favorable property that it simultaneously shows the flame due to its strong density gradients. The $S t=0.01$ case in Figure 3 provides clear evidence that the flame pinch-off and flame island burn-out events are significant sources of sound. The detailed mechanisms by which these events generate sound warrants further investigation, and is the subject of other works by the group (e.g. Talei et al. (2011)).

The ratio of the acoustic energy fluxes leaving and entering the domain can be defined as

$$
\frac{A_{\text {out }} \bar{W}_{2 \text { out }}}{A_{\text {in }} \bar{W}_{2 \text { in }}}=\frac{\int_{A_{\text {out }}} \overline{\left(p^{\prime}+\rho_{0} \mathbf{u}_{\mathbf{0}} \cdot \mathbf{u}^{\prime}\right)\left[\mathbf{u}^{\prime}+\left(\rho^{\prime} / \rho_{0}\right) \mathbf{u}_{\mathbf{0}}\right]} \cdot d A}{\int_{A_{\text {in }}} \overline{\left(p^{\prime}+\rho_{0} \mathbf{u}_{\mathbf{0}} \cdot \mathbf{u}^{\prime}\right)\left[\mathbf{u}^{\prime}+\left(\rho^{\prime} / \rho_{0}\right) \mathbf{u}_{\mathbf{0}}\right]} \cdot d A} .
$$

When this ratio is greater then unity, the flame is generating more sound than is incident from the inlet forcing. Interestingly, Figure 4 shows that is the case only for the simulation 

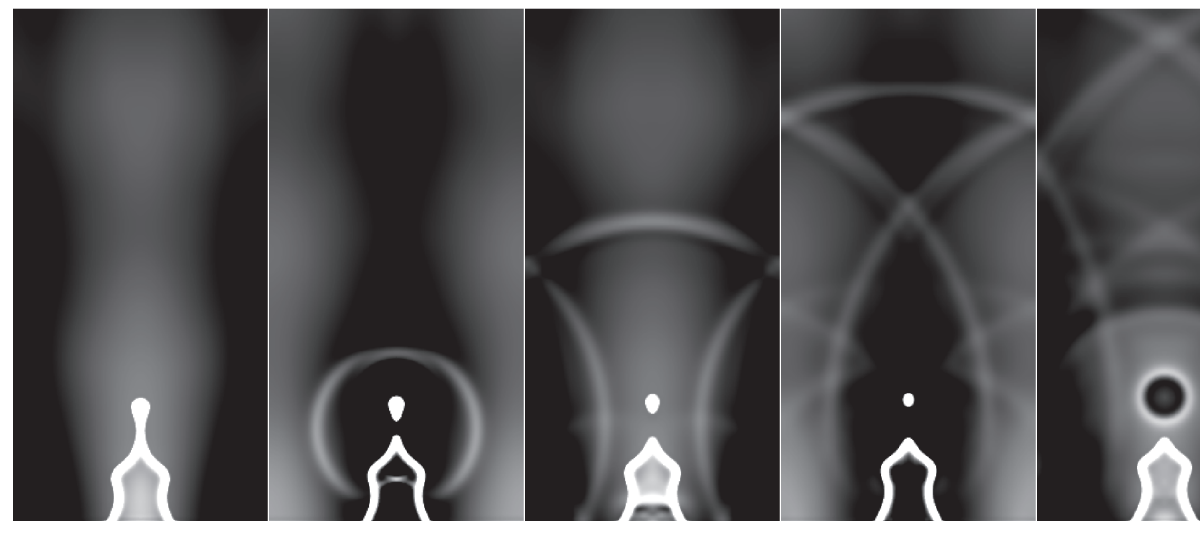

FiguRE 3. Instantaneous dilatation field during the forcing period for $S t=0.02$

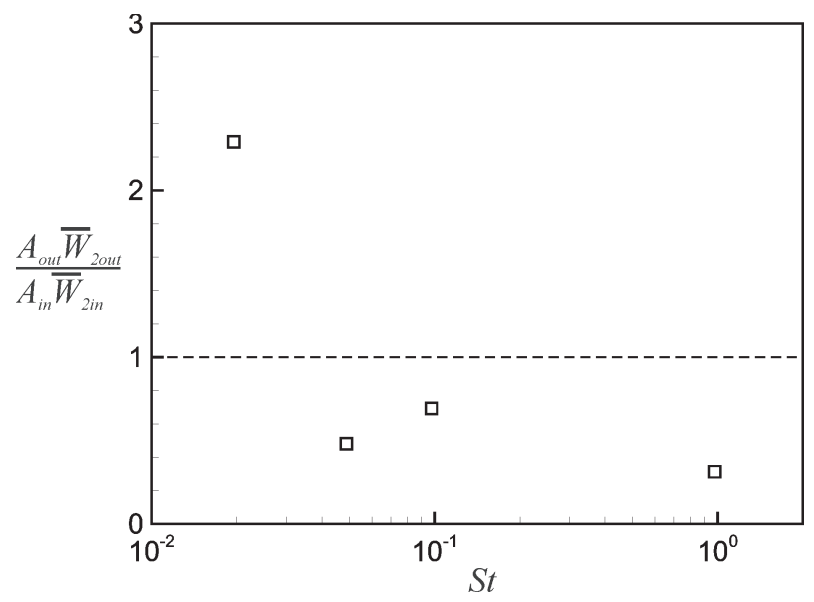

Figure 4. Time averaged acoustic energy flux leaving the domain divided by that entering versus $S t$

performed at $S t=0.02$, which corresponds to the case shown in Figure 3 featuring strong annihilation events. Similar annihilation events do not occur at the other forcing frequencies studied, and plots of the dilation at these frequencies do not reveal acoustic sources within the domain, in contrast to Figure 3.

The time averaged exact disturbance energy density, flux and source terms defined relative to an unsteady base flow in Appendix A are now used to examine the flow further. It was argued in Section 3.3 that the exact disturbance energy flux should become the 
classical acoustic energy flux in some appropriately defined far-field. Figure 5 shows these two fluxes averaged along streamwise and transverse lines across the entire computational domain for two forcing frequencies. It can be seen that these two fluxes become equal just outside the flame, which on average extends in the streamwise and transverse directions to approximately $x / L=4$ and $y / L=1$ respectively. This demonstrates that the far-field defined above is reached well within the computational domain in both cases. The volume integrated and time averaged source terms can now be interpreted as acoustic sources, as per equations 3.3 and A 10, with of course the latter equation appropriate for the present test case.

The time averaged and volume integrated forms of the source terms defined in equation A 10 are shown in Figure 6 versus $S t$. The source terms $\bar{D}_{s}, \bar{D}_{Q}$ and $\bar{D}_{Q^{*}}$ are the largest terms in this flow, and are of similar magnitude. (The source terms $\bar{D}_{\zeta}$ and $\bar{D}_{\psi *}$ are always of order less than unity and are not shown.) Figure 6 therefore shows that several terms other than the Rayleigh term are important, demonstrating that the energetics of sound generation in these simulations cannot be examined by considering the Rayleigh source term alone.

The exact source term $\bar{D}_{Q}=\overline{T^{\prime} Q^{\prime}}$ defined in equation A 10 contains contributions from thermal conduction, species diffusion and viscous dissipation in addition to the heat release by combustion. As such, it can be decomposed into exact and second order source terms concerning only heat release and thermal conduction effects. Figure 7 shows these terms versus $S t$, demonstrating that the flame's heat release is not the only significant part of this exact source term. Further, mean flow effects are significant, as evidenced by the volume integral of the term $-\bar{\omega} \overline{T^{\prime 2}} / \bar{T}^{2}$. Indeed, this term is one that shows that sound can be created or destroyed in the absence of any unsteady heat addition provided 


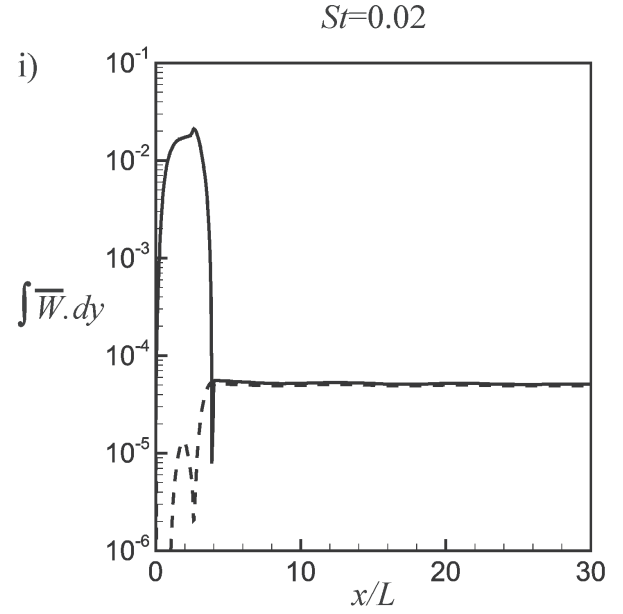
$S t=1$
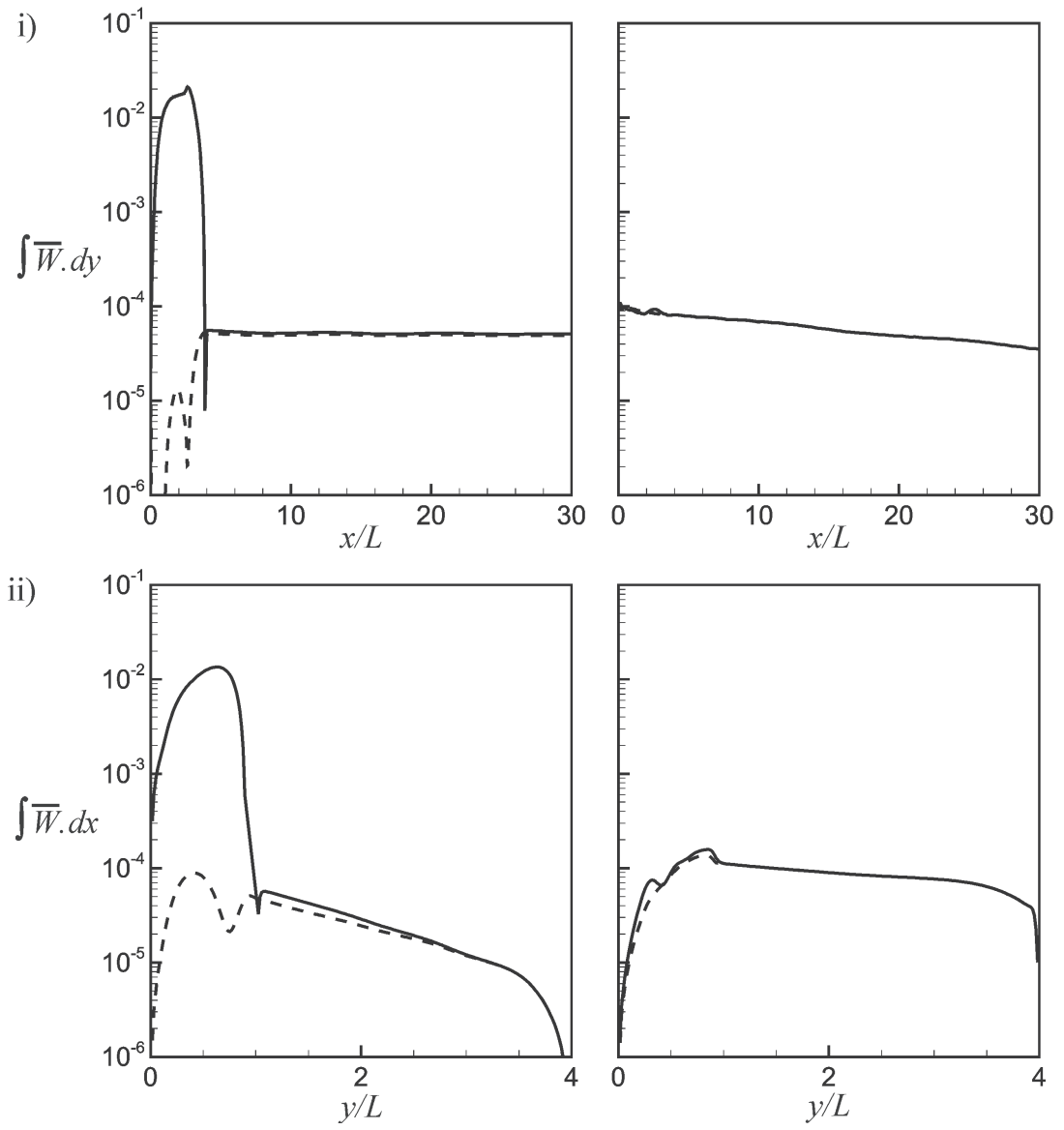

FIgURE 5. Area and time averaged exact disturbance and acoustic energy fluxes for $S t=0.02$ and $S t=1$; i) $\int_{0}^{4 L} \overline{\mathbf{W}} \cdot \mathbf{d y}$ (solid line) and $\int_{0}^{4 L} \overline{\mathbf{W}}_{2}$.dy (dashed line) versus $x$, ii) $\int_{0}^{32 L} \overline{\mathbf{W}} \cdot \mathbf{d x}$ (solid line) and $\int_{0}^{32 L} \overline{\mathbf{W}}_{2} . \mathbf{d x}$ (dashed line) versus $y$. The terms $\mathbf{W}$ and $\mathbf{W}_{\mathbf{2}}$ are from equation A 8 and Cantrell \& Hart (1964) (equation A 10) respectively.

that there is a mean flow. This is examined in detail in the related works Karimi et al. (2008); Nicoud \& Wieczorek (2009); Karimi et al. (2010).

\section{Conclusions}

An analysis of the energy transported by the disturbances in gaseous combustion has been presented. This extended the previous work of Myers [J. Fluid Mech. 226 (1991) 383- 


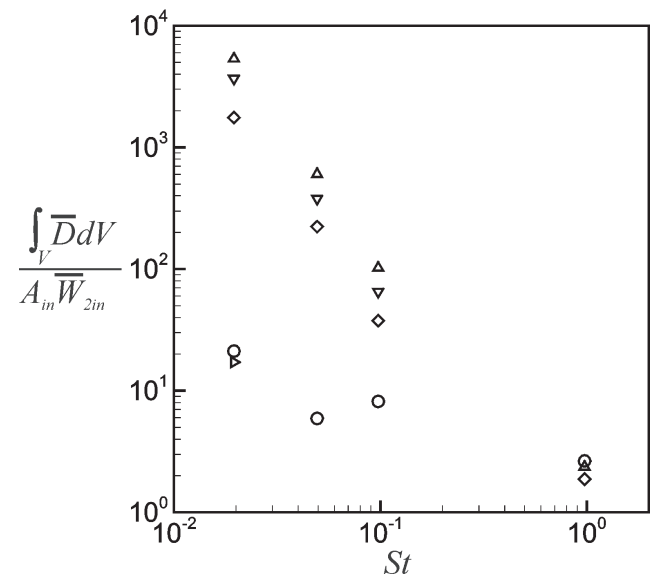

FiguRE 6 . Volume integrated and time averaged exact source terms normalised by $A_{\text {in }} \bar{W}_{2 i n}$ versus $S t, \overline{D_{s}}(\triangle),-\overline{D_{Q}}(\nabla),-\bar{D}_{Q^{*}}^{-}(\diamond),-\overline{D_{\psi}}(\triangleright)$ and $\overline{D_{Y}}(\circ)$.

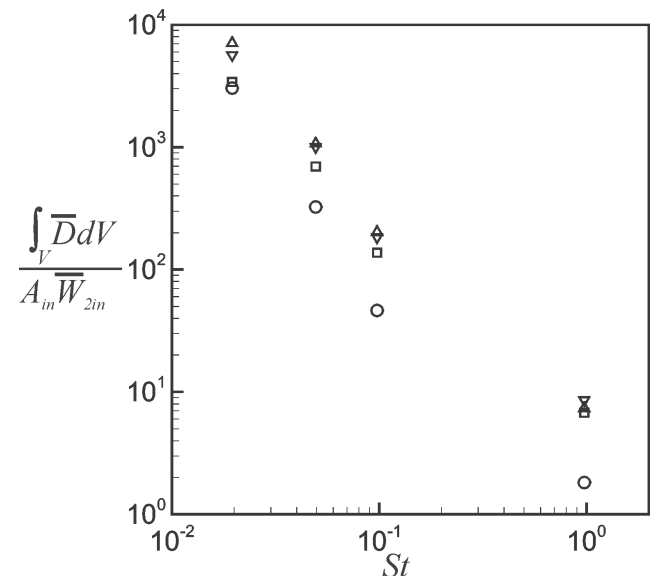

FIgURE 7. Particular time averaged and volume integrated source terms normalised by $A_{i n} \bar{W}_{2 i n}$ versus St: exact heat release $T^{\prime}(\omega / T)^{\prime}(\square)$, exact thermal conduction $T^{\prime}(-\nabla \cdot(\lambda \nabla T) / T)^{\prime}(\triangle)$, second order unsteady heat release $\omega^{\prime} T^{\prime} / \bar{T}(\nabla)$, second order steady heat release $-\bar{\omega} T^{\prime 2} / \bar{T}^{2}(\circ)$. 400] by including multiple species and finite rate chemical reaction, and so derived exact and second order equations describing the transport of disturbance energy in gaseous combustion.

The so-called 'Rayleigh source term' was then derived from these general equations of combusting gas motion. This term was shown to become the sole acoustic source term for flows that feature small disturbances that are irrotational, non-diffusive (in terms of 
species, momentum and energy), homentropic, without mean flow and at chemical equilibrium. In this case the energy density and flux terms attain their classical (zero mean flow) formulation. Further, for non-diffusive, non-zero mean flows at chemical equilibrium, a second order source term proportional to the mean heat release was found. This term can destructively interfere with the Rayleigh term.

Following the approach of Doak [J. Sound Vib. 131 (1989) 67-90], the extended disturbance energy conservation equation was then used to relate the time and area averaged far-field sound from a jet flow to the volume integral of the time average of the exact disturbance energy source terms. A reasonable definition of the far-field was then argued to be one in which entropy and mass fraction disturbances could be neglected. In this case, the relative contribution of each source term to the averaged far-field sound could be compared given complete knowledge of the source region. This approach may be complimentary to more usual analytic approaches in aeroacoustics that use acoustic analogies.

Closure of the exact and second order energy budgets were then performed on a series of aeroacoustic Direct Numerical Simulations (DNS) of a forced, low Mach number, laminar, premixed flame. These balances showed that several source terms were significant, including terms involving mean flow effects and the entropy field. This demonstrates that the energetics of sound generation cannot be examined by considering the Rayleigh source term alone.

The financial support from the Australian Research Council, the Centre Européen de Recherche et de Formation Avancée en Calcul Scientifique (CERFACS), Toulouse and Stanford University is very gratefully acknowledged, as is CERFACS' permission to use the code NTmix. 


\section{Appendix A. Disturbance energy corollary around an unsteady base}

\section{flow}

Around an unsteady base flow, equations 2.12 to 2.16 have time average solution,

$$
\begin{aligned}
\boldsymbol{\nabla} \cdot \overline{\mathbf{m}} & =0, \\
\sum_{k=1}^{n-1}\left[\nabla \cdot\left(\overline{\mathbf{m}} \bar{Y}_{k}\right)+\nabla \cdot\left(\overline{\mathbf{m}^{\prime} Y_{k}^{\prime}}\right)\right. & \left.=\bar{\omega}_{k}-\nabla \cdot\left(\overline{\rho \mathbf{V}_{\mathbf{k}} Y_{k}}\right)\right], \\
\bar{\zeta}+\nabla \bar{H}-\bar{T} \nabla \bar{s}-\left(\overline{T^{\prime} \nabla s^{\prime}}\right) & =\bar{\psi}+\bar{\psi}^{*}, \\
\nabla \cdot(\overline{\mathbf{m}} \bar{H})+\nabla \cdot\left(\overline{\mathbf{m}^{\prime} H^{\prime}}\right)-\overline{\mathbf{m}} \cdot \bar{\psi}-\left(\overline{\mathbf{m}^{\prime} \cdot \psi^{\prime}}\right) & =\bar{T} \bar{Q}+\left(\overline{T^{\prime} Q^{\prime}}\right), \\
\nabla \cdot(\overline{\mathbf{m}} \bar{s})+\nabla \cdot\left(\overline{\mathbf{m}^{\prime} s^{\prime}}\right) & =\bar{Q}+\bar{Q}^{*} .
\end{aligned}
$$

Here, ()$\left.^{\prime}=()-\overline{(}\right)$ is the disturbance and $\overline{()}$ is the time averaged value.

Solutions to equations A 1 to A 5 now become the base flow. Undertaking the same algebra as that detailed in Section 2.2, this results in an exact conservation equation for the disturbance energy in a slightly different form,

$$
\frac{\partial E}{\partial t}+\nabla \cdot \mathbf{W}=D
$$

In equation A 6, the disturbance energy density $E$ and flux vector $\mathbf{W}$ terms are now

$$
E=\rho\left(H^{\prime}-\bar{T} s^{\prime}\right)-\overline{\mathbf{m}} \cdot \mathbf{u}^{\prime}-p^{\prime}-\rho \sum_{k=1}^{n-1}\left[g_{k} Y_{k}^{\prime}-\left(\overline{g_{k}^{\prime} Y_{k}^{\prime}}\right)\right],
$$

and

$$
\mathbf{W}=\mathbf{m}^{\prime}\left(H^{\prime}-\bar{T} s^{\prime}\right)+\overline{\mathbf{m}} T^{\prime} s^{\prime}+\left(\overline{\mathbf{m}^{\prime} H^{\prime}}\right)-T\left(\overline{\mathbf{m}^{\prime} s^{\prime}}\right)+\mathbf{m}\left(\overline{T^{\prime} s^{\prime}}\right)
$$

The exact source term $D$ is again

$$
D=D_{\xi}+D_{s}+D_{Q}+D_{Q^{*}}+D_{\psi}+D_{\psi^{*}}+D_{Y_{k}}
$$

where now

$$
\begin{aligned}
& D_{\xi}=-\mathbf{m}^{\prime} \cdot \boldsymbol{\zeta}^{\prime}-\left(\overline{\mathbf{m}^{\prime} \cdot \boldsymbol{\zeta}^{\prime}}\right), \\
& D_{s}=-\left(\mathbf{m}^{\prime} s^{\prime}\right) \cdot \nabla \bar{T}+\overline{\mathbf{m}} \cdot\left(s^{\prime} \boldsymbol{\nabla} T^{\prime}\right)-\left(\overline{\mathbf{m}^{\prime} s^{\prime}}\right) \cdot \nabla T+\mathbf{m} \cdot\left(\overline{s^{\prime} \nabla T^{\prime}}\right),
\end{aligned}
$$




$$
\begin{aligned}
D_{Q} & =T^{\prime} Q^{\prime}+\left(\overline{T^{\prime} Q^{\prime}}\right), \\
D_{Q^{*}} & =T^{\prime} Q^{* \prime}+\left(\overline{T^{\prime} Q^{* \prime}}\right), \\
D_{\psi} & =\mathbf{m}^{\prime} \cdot \boldsymbol{\psi}^{\prime}+\left(\overline{\mathbf{m}^{\prime} \cdot \boldsymbol{\psi}^{\prime}}\right), \\
D_{\psi^{*}} & =\mathbf{m}^{\prime} \cdot \boldsymbol{\psi}^{* \prime}+\left(\overline{\mathbf{m}^{\prime} \cdot \boldsymbol{\psi}^{* \prime}}\right), \\
D_{Y_{k}} & =g^{\prime} \boldsymbol{\nabla} \cdot \mathbf{m}^{\prime}+\left(\overline{g^{\prime} \boldsymbol{\nabla} \cdot \mathbf{m}^{\prime}}\right)+\sum_{k=1}^{n-1}\left[g_{k}^{\prime} \Omega_{k}^{\prime}+\left(\overline{g_{k}^{\prime} \Omega_{k}^{\prime}}\right)\right] .
\end{aligned}
$$

As discussed in Section 3.3, application of Gauss' law to the time average of equation A 6 and defining the far-field in the same way, results in the following expression for the time and area averaged, far-field sound.

$$
\int_{A} \overline{\left(p^{\prime}+\rho_{0} \mathbf{u}_{\mathbf{0}} \cdot \mathbf{u}^{\prime}\right)\left[\mathbf{u}^{\prime}+\left(\rho^{\prime} / \rho_{0}\right) \mathbf{u}_{\mathbf{0}}\right]} \cdot d \mathbf{A}=\int_{V}\left(\bar{D}_{\xi}+\bar{D}_{s}+\bar{D}_{Q}+\bar{D}_{Q^{*}}+\bar{D}_{\psi}+\bar{D}_{\psi^{*}}+\bar{D}_{Y_{k}}\right) d V
$$

where

$$
\begin{gathered}
\bar{D}_{\xi}=-\overline{\mathbf{m}^{\prime} \cdot \zeta^{\prime}}, \bar{D}_{s}=-\left(\overline{\mathbf{m}^{\prime} s^{\prime}}\right) \cdot \nabla \bar{T}+\overline{\mathbf{m}} \cdot\left(\overline{s^{\prime} \nabla T^{\prime}}\right), \bar{D}_{Q}=\overline{T^{\prime} Q^{\prime}}, \bar{D}_{Q^{*}}=\overline{T^{\prime} Q^{*^{\prime}}} \\
\bar{D}_{\psi}=\overline{\mathbf{m}^{\prime} \cdot \boldsymbol{\psi}^{\prime}}, \bar{D}_{\psi^{*}}=\overline{\mathbf{m}^{\prime} \cdot \boldsymbol{\psi}^{* \prime}}, \bar{D}_{Y_{k}}=\overline{g^{\prime} \boldsymbol{\nabla} \cdot \mathbf{m}^{\prime}}+\sum_{k=1}^{n-1} \overline{g_{k}^{\prime} \Omega_{k}^{\prime}} .
\end{gathered}
$$

Equation A 6 has several new terms that did not appear in equation 2.22 due to the differing choices of base flow. Importantly, when time averaged, all of these new terms duplicate terms that have already appeared in equation 2.22 , giving a relatively simple form to equation A 10.

\section{Appendix B. Second order energy corollary around a steady base}

flow

Equation 2.23 states the exact energy density,

$$
E=\rho\left[H^{\prime}-T_{0} s^{\prime}\right]-\mathbf{m}_{\mathbf{0}} \cdot \mathbf{u}^{\prime}-p^{\prime}-\rho \sum_{k=1}^{n-1} g_{k 0} Y_{k}^{\prime},
$$




$$
\begin{aligned}
= & \rho e+\frac{1}{2} \rho \mathbf{u} \cdot \mathbf{u}-\rho e_{0}-\frac{\rho}{\rho_{0}} p_{0}-\frac{1}{2} \rho \mathbf{u}_{\mathbf{0}} \cdot \mathbf{u}_{\mathbf{0}}-\rho T_{0} s^{\prime}-\mathbf{m}_{\mathbf{0}} \cdot \mathbf{u}^{\prime}-p_{0} \\
& -\rho \sum_{k=1}^{n-1} g_{k 0} Y_{k}^{\prime},
\end{aligned}
$$

where $e$ is the sensible internal energy. The term $\rho e$ is then expanded as a $(n+1)$ dimensional Taylor series in $\rho^{\prime}, s^{\prime}$ and $Y_{1}^{\prime} \ldots Y_{n-1}^{\prime}$ to second order,

$$
\begin{aligned}
\rho e & =\rho_{0} e_{0}+\left(\frac{\partial \rho e}{\partial \rho}\right)_{0} \rho^{\prime}+\left(\frac{\partial \rho e}{\partial s}\right)_{0} s^{\prime}+\sum_{k=1}^{n-1}\left(\frac{\partial \rho e}{\partial Y_{k}}\right)_{0} Y_{k}^{\prime} \\
& +\left(\frac{\partial^{2} \rho e}{\partial \rho^{2}}\right)_{0} \frac{\rho^{\prime 2}}{2}+\left(\frac{\partial^{2} \rho e}{\partial s^{2}}\right)_{0} \frac{s^{\prime 2}}{2}+\sum_{k=1}^{n-1}\left(\frac{\partial^{2} \rho e}{\partial Y_{k}^{2}}\right)_{0} \frac{Y_{k}^{\prime 2}}{2}+\left(\frac{\partial^{2} \rho e}{\partial \rho \partial s}\right)_{0} \rho^{\prime} s^{\prime} \\
& +\sum_{k=1}^{n-1}\left[\left(\frac{\partial^{2} \rho e}{\partial \rho \partial Y_{k}}\right)_{0} \rho^{\prime} Y_{k}^{\prime}+\left(\frac{\partial^{2} \rho e}{\partial s \partial Y_{k}}\right)_{0} s^{\prime} Y_{k}^{\prime}+\sum_{j=1}^{n-1}\left(\frac{\partial^{2} \rho e}{\partial Y_{j} \partial Y_{k}}\right)_{0} Y_{j \neq k}^{\prime} Y_{k}^{\prime}\right]
\end{aligned}
$$

Evaluation of the partial derivatives in B 2 first requires derivation of several exact differentials. Those derivatives not involving $Y_{k}$ are presented in Myers (1991) and are true at chemical equilibrium, whilst derivatives involving $Y_{k}$ require use of non-equilibrium differentials given in Appendix C. Substitution of equation B 2 into equation B 1 then yields the second order energy density,

$$
\begin{aligned}
E_{2} & =\frac{p^{\prime 2}}{2 \rho_{0} c_{0}^{2}}+\frac{1}{2} \rho_{0} u^{\prime 2}+\rho^{\prime} \mathbf{u}_{0} \cdot \mathbf{u}^{\prime}+\frac{\rho_{0} T_{0} s^{\prime 2}}{2 c_{p 0}} \\
& -\frac{p^{\prime}}{\rho_{0} c_{0}^{2}} \sum_{k=1}^{(n-1)}\left[\frac{\rho_{0} R T_{0}}{W_{k 0}}+\frac{p_{0}}{c_{v 0} T_{0}}\left(g_{k 0}-e_{k 0}\right)\right] Y_{k}^{\prime}, \\
& +\frac{1}{2 \rho_{0} c_{0}^{2}} \sum_{j=1}^{(n-1)} \sum_{k=1}^{(n-1)}\left[\frac{\rho_{0} R T_{0}}{W_{j 0}}+\frac{p_{0}}{c_{v 0} T_{0}}\left(g_{j 0}-e_{j 0}\right)\right]\left[\frac{\rho_{0} R T_{0}}{W_{k 0}}+\frac{p_{0}}{c_{v 0} T_{0}}\left(g_{k 0}-e_{k 0}\right)\right] Y_{j}^{\prime} Y_{k}^{\prime} \\
& +\sum_{j=1}^{(n-1)} \sum_{k=1}^{(n-1)} \frac{\rho_{0}}{c_{v 0}}\left(c_{p j 0}-c_{v j 0}+\frac{R}{W_{j 0}}-s_{j 0}\right)\left[\left(g_{j 0}-e_{j 0}\right)+\frac{R}{Y_{j 0} W_{j 0}}\right] Y_{j \neq k}^{\prime} Y_{k}^{\prime} \\
& +\sum_{k=1}^{(n-1)} \frac{\rho_{0}}{2 c_{v 0}}\left(c_{p k 0}-c_{v k 0}+\frac{R}{W_{k 0}}-s_{k 0}\right)\left[\left(g_{k 0}-e_{k 0}\right)+\frac{R}{Y_{k 0} W_{k 0}}\right] Y_{k}^{\prime 2}, \\
& +\sum_{k=1}^{(n-1)}\left[\frac{R T_{0}}{W_{k 0}}+\left(\gamma_{0}-1\right)\left(g_{k 0}-e_{k 0}\right)\right] \rho^{\prime} Y_{k}^{\prime}, \\
& +\sum_{k=1}^{(n-1)} \frac{\rho_{0}}{c_{v 0}}\left(g_{k 0}-e_{k 0}\right) s^{\prime} Y_{k}^{\prime} .
\end{aligned}
$$


The exact flux vector (equation 2.24) is already second order in the disturbances. It can also be expressed in form which more obviously can reduce to the acoustic energy flux by making use of Gibbs' equation in terms of the enthalpy of the mixture (e.g. Williams $(1985))$

$$
d h=T d s+\frac{1}{\rho} d p+\sum_{k=1}^{n} g_{k} d Y_{k}
$$

The flux vector is then

$$
\begin{aligned}
\mathbf{W}_{\mathbf{2}} & =\mathbf{m}^{\prime}\left(H^{\prime}-T_{0} s^{\prime}\right)+\mathbf{m}_{\mathbf{0}} T^{\prime} s^{\prime} \\
& =\left(p^{\prime}+\rho_{0} \sum_{k=1}^{n-1} g_{k 0} Y_{k}^{\prime}+\rho_{0} \mathbf{u}_{\mathbf{0}} \cdot \mathbf{u}^{\prime}\right)\left(\mathbf{u}^{\prime}+\frac{\rho^{\prime}}{\rho_{0}} \mathbf{u}_{\mathbf{0}}\right)+\mathbf{m}_{\mathbf{0}} T^{\prime} s^{\prime},
\end{aligned}
$$

where the term containing $Y_{k}^{\prime}$ can be considered as a flux of energy associated with mass fraction disturbances.

Similarly, the exact source term (equation 2.25) is already second order in the disturbances. Neglecting the viscous stress, dissipation, heat conduction and species diffusion terms, it can be further simplified as follows.

$$
\begin{aligned}
D_{2} & =\rho_{0} \mathbf{u}_{\mathbf{0}} \cdot\left(\boldsymbol{\xi}^{\prime} \times \mathbf{u}^{\prime}\right)+\rho^{\prime} \mathbf{u}^{\prime} \cdot\left(\boldsymbol{\xi}_{\mathbf{0}} \times \mathbf{u}_{\mathbf{0}}\right) \\
& -s^{\prime} \mathbf{m}^{\prime} \cdot\left(\boldsymbol{\nabla} T_{0}\right)+s^{\prime} \mathbf{m}_{\mathbf{0}} \cdot\left(\boldsymbol{\nabla} T^{\prime}\right) \\
& +\left(\frac{\omega^{\prime} T^{\prime}}{T_{0}}-\frac{\omega_{0} T^{2}}{T_{0}^{2}}\right) \\
& -\frac{T^{\prime}}{T_{0}} \sum_{k=1}^{n-1}\left(g_{k 0} \omega_{k}^{\prime}+g_{k}^{\prime} \omega_{k 0}\right) \\
& +\mathbf{m}^{\prime} \cdot\left[\sum_{k=1}^{n-1}\left(g_{k}^{\prime} \nabla Y_{k 0}+g_{k 0} \boldsymbol{\nabla} Y_{k}^{\prime}\right)\right] \\
& +g^{\prime} \nabla \cdot \mathbf{m}^{\prime}+\sum_{k=1}^{n-1} g_{k}^{\prime}\left[\omega_{k}^{\prime}-\nabla \cdot\left(\mathbf{m}_{\mathbf{0}} Y_{k}^{\prime}+\mathbf{m}^{\prime} Y_{k 0}\right)\right] .
\end{aligned}
$$




\section{Appendix C. Exact thermodynamic differentials for non-equilibrium chemistry}

Gibbs' equation states an exact differential for the internal energy $e$ in terms of the entropy, density and mass fractions,

$$
d e=T d s+\frac{p}{\rho^{2}} d \rho+\sum_{k=1}^{n} g_{k} d Y_{k}
$$

Since

$$
e=\int_{T_{0}}^{T} c_{v} d T-\frac{R T_{0}}{W}
$$

where $R$ is the ideal gas constant and $W=1 /\left(\sum_{k=1}^{n} Y_{k} / W_{k}\right)$ is the molecular mass, de can also be written as

$$
d e=c_{v} d T+\sum_{k=1}^{n} e_{k} d Y_{k} .
$$

Equating equations $\mathrm{C} 1$ and $\mathrm{C} 3$ then gives an exact differential for the temperature

$$
d T=\frac{1}{c_{v}}\left[T d s+\frac{p}{\rho^{2}} d \rho+\sum_{i=1}^{n}\left(g_{k}-e_{k}\right) d Y_{k}\right] .
$$

Similar working yields exact differentials for the pressure, enthalpy of the mixture and the Gibb's free energy of species $k$,

$$
\begin{aligned}
d p & =\frac{p}{c_{v}} d s+\frac{\gamma p}{\rho} d \rho+\sum_{k=1}^{n}\left[\frac{\rho R T}{W_{k}}+\frac{\rho R}{W c_{v}}\left(g_{k}-e_{k}\right)\right], \\
d h & =T d s+\frac{\gamma p}{\rho^{2}} d \rho+\sum_{k=1}^{n}\left[\frac{R T}{W_{k}}+\gamma g_{k}-(\gamma-1) e_{k}\right] d Y_{k}, \\
d g_{k} & =\left(c_{p k}-c_{v k}+\frac{R}{W_{k}}-s_{k}\right) \frac{T}{c_{v}} d s \\
& +\frac{R T}{\rho W}\left[\frac{W}{c_{v}}\left(c_{p k}-c_{v k}+\frac{R}{W_{k}}-s_{k}\right)+\frac{W}{W_{k}}\right] d \rho \\
& +\frac{1}{c_{v}}\left(c_{p k}-c_{v k}+\frac{R}{W_{k}}-s_{k}\right) \sum_{k=1}^{n}\left[\left(g_{k}-e_{k}\right)+\frac{R}{Y_{k} W_{k}}\right] d Y_{k} .
\end{aligned}
$$


BAum, M. 1994 Etude de l'allumage et de la structure des flammes turbulentes. PhD thesis, Ecole Centrale Paris.

Bloxsidge, G. J., Dowling, A. P., Hooper, N. \& Langhorne, P. J. 1988 Active control of reheat buzz. AIAA Journal 26 (7), 783-790.

Bourlioux, A., Cuenot, B. \& Poinsot, T. 2000 Asymptotic and numerical study of the stabilization of diffusion flames by hot gas. Combustion and Flame 120 (1-2), 143-59.

CAndel, S. 2002 Combustion dynamics and control: progress and challenges. Proc. Combust. Inst. 29, 1-28.

CANTREll, R. H. \& HART, R. W. 1964 Interaction between sound and flow in acoustic cavities: mass, momentum and energy considerations. Journal of the Acoustical Society of America 36, $697-706$.

Chu, B. T. 1956 Stability of systems containing a heat source - the Raleigh Criterion. NACA Research memorandum (NACA RM 56D27).

Chu, B. T. 1965 On the energy transfer to small disturbances in fluid flow (Part 1). ACTA Mechanica 1, 215-234.

Colonius, T., Lele, S. K. \& Moin, P. 1997 Sound generation in a mixing layer. J. Fluid Mech. 330, 375-409.

Corjon, A. \& Poinsot, T. 1995 A model to define aircraft separations due to wake vortex encounter. In 13th AIAA Applied Aerodynamics Conference. AIAA paper 95-1776., pp. $117-124$.

Corjon, A. \& Poinsot, T. 1997 Behavior of wake vortices near ground. AIAA Journal 35 (5), 849-855.

Cuenot, B., Bedet, B. \& Corjon, A. 1997 NTMIX3D user's guide manual, Preliminary Version 1.0 .

Culick, F. E. C. 2001 Dynamics of combustion systems: fundamentals, acoustics and control. In Active control of engine dynamics, pp. 89-206. NATO / Von Karman Institute.

DoAK, P. E. 1989 Momentum potential-theory of energy flux carried by momentum fluctuations. J. Sound Vib. 131 (1), 67-90. 
Dowling, A. P. 1997 Acoustics of unstable flows. Theoretical and Applied Mechanics X, 171186.

Dowling, A. P. \& Stow, S. R. 2003 Acoustic analysis of gas turbine combustors. AIAA Journal of Propulsion and Power 19 (5), 751-764.

Flandro, G. A. 1985 Energy balance analysis of nonlinear combustion instability. AIAA Journal of Propulsion and Power 1 (3), 210-221.

Goldstein, M. E. 2005 On identifying the true sources of aerodynamic sound. J. Fluid Mech. 526, 337-347.

Karimi, N., Brear, M., Jin, S. \& Monty, J. 2009 Linear and non-linear forced response of a conical, ducted, laminar premixed flame. Combustion and Flame 156, 2201-2212.

Karimi, N., Brear, M. \& Moase, W. 2008 Acoustic and disturbance energy analysis of a flow with heat communication. Journal of Fluid Mechanics 597, 67-89.

Karimi, N., Brear, M. \& Moase, W. 2010 On the interaction of sound with steady heat communicating flows. Journal of Sound and Vibration 329, 4705-4718.

Lodato, G., Domingo, P. \& Vervisch, L. 2008 Three-dimensional boundary conditions for direct and large-eddy simulation of compressible viscous flows. Journal of Computational Physics 227 (10), 5105-5143.

LORD RAYLEIGH 1878 The explanation of certain acoustical phenomena. Nature 18, 319-321.

Morfey, C. L. 1971 Acoustic energy in non-uniform flows. J. Sound Vib. 14 (2), 159-170.

Myers, M. K. 1991 Transport of energy by disturbances in artitrary steady flows. J. Fluid Mech. 226, 383-400.

Nicoud, F. \& Poinsot, T. 2005 Thermoacoustic instabilities: Should the Raleigh Criterion be extended to include entropy changes? Combustion and Flame 142, 153-159.

Nicoud, F. \& Wieczorek, K. 2009 About the zero mach number assumption in the calculation of thermoacoustic instabilities. International Journal of Spray and Combustion Dynamics $1(1), 67-111$.

Pierce, A. D. 1981 Acoustics - An Introduction to its Physical Principles and Applications. McGraw-Hill. 
Poinsot, T. J. \& Lele, S. 1992 Boundary conditions for direct simulations of compressible viscous flows. Journal of Computational Physics 101 (1), 104-129.

Poinsot, T. J. \& Veynante, D. 2001 Theoretical and numerical combustion. R.T. Edwards.

Putnam, A. A. 1971 Combustion driven oscillations in industry. American Elsevier.

Schuller, T., Durox, D. \& CANDEl, S. 2003 A unified model for the prediction of laminar flame transfer functions: comparisons between conical and v-flame dynamics. Combustion and Flame 134, 21-34.

Schwarz, A. \& JANiCKA, J. 2009 Combustion noise. Springer.

Talei, M., Brear, M. \& Hawkes, E. 2011 Sound generation by premixed flame annihilation. Journal of Fluid Mechanics, in press .

Talei, M., Brear, M. \& Hawkes, E. 2012 A parametric study of sound generation by laminar premixed flame annihilation. Combustion and Flame, in press .

Williams, F. 1985 Combustion Theory. The Fundamental Theory of Chemically Reacting Flow Systems. Addison Wesley.

Yoo, C., Wang, Y., Trouve, A. \& Im, H. 2005 Characteristic boundary conditions for direct simulations of turbulent counterflow flames. Combustion Theory and Modelling 9 (4), 617646. 